\title{
Management of Breast Cancer in Nepal
}

\author{
Singh YP,' Sayami $P^{1}$ \\ 'Department of Surgery, Institute of Medicine, TUTH, Kathmandu, Nepal.
}

\begin{abstract}
Breast cancer is the second most common malignancy among women in Nepal. It is more common in young premenopausal women. Breast cancer continues to increase in incidence due to lifestyle changes in Nepalese women despite constant remarkable development in the management of this disease over the past three decades. Breast cancer was diagnosed solely clinically and surgery was the only treatment option until fifty years ago. Multidisciplinary approach has been adopted for diagnosis and treatment of breast cancer in Nepal. Imaging is required for the diagnosis, appropriate treatment decision and proper follow up. Treatment modality depends upon the extent of the disease and tumor biology. However, there is a strong need for standard guidelines for the proper management of breast cancer in Nepal so that surgeries, chemotherapy, hormone therapy and radiotherapy are standardized in the country. Palliative care has been initiated to provide to some patients with metastatic breast cancer recently.
\end{abstract}

The breast cancer management in Nepal is a little different when compared with the centers in the developed countries. The reasons are socioeconomic status, lack of education and lack of facilities. Although cancer care is on the rise in Nepal, the optimal facility for centers managing breast cancer has to be improved significantly.Cancer education, screening and early detection are the key elements to influence the diagnosis, treatment and prognosis of breast cancer in Nepal. Breast cancer awareness and clinical breast examination are important tools for early detection in our resource limited context. Breast cancer can be cured in majority of the cases if diagnosed in early stages. This review will focus on relevant patient data along with future recommendation regarding breast cancer treatment in Nepal.

Key Words: Breast cancer, cancer education, chemotherapy, imaging, radiotherapy, surgery

\section{INTRODUCTION}

Breast cancer is a major health problem to affect women worldwide. In Nepal also, as breast cancer continues to increase, all concerned people must act promptly to control this disease. Although there are a few comprehensive cancer centers in Nepal, breast cancer is managed at other centers with limited resources frequently. There is no uniformity in the guidelines of managing all breast cancer. The management of the breast carcinoma patients has undergone revolutionary changes over the past three decades worldwide. Some positive changes can be appreciable in Nepal too. The objective of this article is to analyze all the data for managing breast cancer in Nepal. A relevant search was made using PubMed, entering Breast cancer in Nepal as search sentence and information distilled and presented. In this article, we discuss about breast 
cancer incidence, detection and management, focusing on areas of clinical practice that has evolved in Nepal or where emerging evidence supports future directions in practice.

\section{Incidence:}

There is a marked geographical variation in the incidence of breast carcinoma worldwide. In the West (developed countries), the incidence of breast cancer is more than 1000 per million whereas in developing countries it is low (less than 200 per million women). However, cancer mortality is higher in developing countries than in the developed countries. ${ }^{1}$ There is a lack of population-based cancer registry in Nepal. However, it is encouraging to note that some promising efforts have been initiated at least in terms of hospital based registry. ${ }^{2,3}$ Breast cancer accounts for $6 \%$ of all cancers in Nepal. ${ }^{4} \mathrm{~A}$ recent multi-institution hospital-based cancer incidence data revealed breast cancer as the second most common cancer among women in Nepal. ${ }^{2}$ The commonest age group of women with breast cancer in Nepal is 40-50 years. One of the most remarkable differences is higher incidence of younger breast cancer women $(<50 \mathrm{yr})$ in our part of the world.

\section{Risk Factors:}

The effect of endogenous hormones is a major contributing factor for the etiology of breast cancer. Among hormonal factors, reproductive history such as nulliparity, early age at menarche, late age at menopause and late age at first full-term pregnancy is associated with an increased risk. ${ }^{5}$ However, Nepalese breast cancer is commonly seen in young age with late menarche, early first full-term pregnancy, a long duration of lactation.6, 7 A family history positive for breast cancer produces an increased risk. ${ }^{5}$ In young patients, genetically inherited susceptibility to breast cancer is well described. Family history of breast carcinoma in first degree relatives is rare in Nepal. ${ }^{6,}{ }^{7}$ None of the known reproductive factors can adequately explain the early occurrence of premenopausal Nepalese breast cancer. Due to modernization and development in urban areas of Nepal, there has been a large influx of people into these areas. Life style changes like dietary habits, lack of physical exercise, less breast feeding by mothers, are seen more frequently in urban women. These factors might contribute to an increased risk of breast cancer.

\section{Early Detection and Screening:}

Patients with early breast cancers are usually asymptomatic. Early detection and screening programs are established in order to reduce the breast cancer mortality. Breast Self-Examination (BSE) and clinical breast examination are the key steps for the early detection of breast cancer in resource limited countries like Nepal. BSE is an inexpensive tool for creating breast cancer awareness although it does not provide survival benefit. If a woman practices BSE regularly, she is not only conscious about breast cancer but also about her health in general. The evidence shows that screening mammography and proper examination of breasts can be useful in reducing breast cancer mortality. ${ }^{8}$ However, a population based study from eastern region of Nepal concluded that BSE could be used as an important tool for primary prevention of breast cancer in Nepal. ${ }^{9}$ Clinical breast examination by trained female nurses has been initiated as a pilot screening program recently. At present, breast cancer screening by mammogram is not feasible due to unavailability of this facility at many centers though few early cancers can be detected by mammograms. ${ }^{10}$ High-resolution ultrasound can detect very small lesions, and more reliably differentiates malignant from benign solid masses. ${ }^{11,12}$ However, ultrasound remains unproven as a screening tool, and its potential role in screening is likely to be limited in cases where mammography can not detect lesions in young women with dense breasts. ${ }^{13}$

\section{Presentation:}

The average age of breast cancer is $46.5 \pm 12.8$ years ranging from 25 to 80 years. ${ }^{7}$ The diagnosis of asymptomatic breast cancer is rare in Nepal. Painless breast lump is the commonest presentation (80$90 \%$ ) which is ignored by many women in developing countries including Nepal. The duration of symptoms varies from 8 to 13 months which has decreased to 4 to 5 months recently. ${ }^{6,7}$ More than half of them (60\%) are premenopausal young women ( $<50$ year). The ratio of pre- to post-menopausal breast cancer is 6 to 4 in Nepal whereas it is 4 to 6 in the West. ${ }^{14}$ Nepalese breast cancer cases, particularly premenopausal breast cancer patients, are seen at a young age with late menarche, early first full-term pregnancy, long duration of lactation and a large number of children. ${ }^{7}$ The mean tumor size is $6.1 \pm 2.5 \mathrm{~cm}$ ranging from 1.3 to $20 \mathrm{~cm}$ which has come down to $3.9 \mathrm{~cm}$ recently. ${ }^{7}$ Staging of breast cancer is a part of pretreatment evaluation to assess the extent of the disease and treatment. Although the trend is improving to present early, more than half of our breast cancer patients present in late stage (III or IV). The recent data at our institute reveal carcinoma in situ (1\%), stage I (7\%), stage II (IIA 19\%, IIB 23\%), stage III (IIIA 18\%, IIIB 22\%), and stage IV (10\%).

\section{Imaging:}

Remarkable changes have been seen in breast imaging and diagnostic modalities of breast cancer. Over the year imaging has a key role for accurate diagnosis, appropriate treatment selection and follow-up plan. Mammography is routinely done for all women over the age of 40 years 
Singh et al. Management of Breast Cancer in Nepal

attending symptomatic breast clinics. High-resolution ultrasound is an effective diagnostic tool for focal breast symptoms. Mammogram and breast ultrasound services are available at a few centers in Nepal. Based on one study only $68 \%$ of our breast cancer patients had undergone mammography before surgery. ${ }^{10}$ This may be due to unavailability of mammogram in the center where breast cancer is managed frequently. Breast carcinoma was identified on mammography in $87 \%$ of cases. In the rest of the patients (reported as either normal or benign lesions), the diagnosis was missed in mainly younger and premenopausal groups of women. The diagnostic accuracy for carcinomas of the breast appears to improve when mammography is combined with ultrasonogram even in cases that reveal no evidence of microcalcification or other abnormalities. ${ }^{15}$ Magnetic resonance imaging (MRI) of the breast is not routinely used in the diagnosis. However, it is an important tool for evaluating the extent of the disease (multifocality) where this cannot be reliably excluded on conventional imaging. ${ }^{16}$ Interest in breast $\mathrm{MRI}$ in recent years has largely focused on its role in screening women with inherited mutations of the breast cancer genes (BRCA1 or BRCA2). ${ }^{17}$

\section{Pathology:}

Histocytological diagnosis has a pivotal role for the definitive treatment of breast cancer. The commonest diagnostic modality of breast lump is to perform fine needle aspiration cytology (FNAC) before further evaluation or operative treatment. ${ }^{18}$ FNAC is a popular technique because it is a simple, safe and quick procedure with high sensitivity and specificity. ${ }^{19}$ The accuracy rate of FNAC in diagnosing breast cancer is up to $96 \% .^{20,21}$ Other than morphological prognostic factors, molecular predictive factors are essential to predict response to systemic treatments such as hormone receptors, HER2 and so on. Fortunately this facility is available. Less than $40 \%$ of our breast cancers are hormone receptors (Estrogen and/or Progesterone) positive.$^{10}$ In the West or Japan, the hormone receptor positive rate is more than $60 \% .{ }^{22}$ FNAC is commonly used in addition to clinical examination and mammography for the diagnosis of breast lesions. ${ }^{23}$ Triple test score (physical examination, mammography and fine needle aspiration cytology, each component is designated as benign, suspicious and malignant and rated as 1,2 , and 3, respectively) can be an accurate diagnostic modality to initiate definitive treatment of breast carcinoma specially in our context where frozen section is not routinely available. ${ }^{24}$ Percutaneous core needle biopsy (CNB) has widely replaced cytology obtained by fine-needle aspiration, and is the established method of sampling imagedetected lesions. ${ }^{25} \mathrm{CNB}$ provides more information than FNAC and assists in planning therapy before surgery in women with cancer, as it reliably distinguishes between invasive and in-situ disease, and allows determination of hormone and growth factor receptors. ${ }^{26}$

\section{Surgery:}

The local treatment of breast cancer can be performed in order to achieve a long-term local disease control with minimum local morbidity. Breast conserving surgery (BCS) produces an acceptable cosmetic appearance. ${ }^{27}$ So that less anxiety and depression and improved body image and sexuality when compared with mastectomy. ${ }^{28}$ Moreover, there is no difference in disease outcome between BCS in combined with radiotherapy and mastectomy. ${ }^{29,30}$ The history of breast cancer surgery is as old as the history of surgery in Nepal. Until four decades back radical mastectomy was the main surgical option for breast cancer. The majority of women presenting to the hospital have large breast cancers, which are not suitable for breast conserving surgery. There is slowly changing trend of breast conserving surgery (BCS) in Nepal. The recent trends of BCS at $\mathrm{BPKMCH}$, Bharatpur and our institute (TUTH) are $6 \%$ and $15 \%$, respectively. The majority of them undergo total mastectomy with axillary lymph nodes dissection of level I and II. ${ }^{6}$ Moreover, BCS is not offered in the centers where proper preoperative evaluation facility and postoperative radiotherapy are not available. Axillary lymph node status is the most significant individual prognostic factor in breast cancer patients. ${ }^{31}$ Both adjuvant systemic and locoregional treatments depend upon this factor. Standard management of the axilla has been axillary node dissection. However this procedure is associated with significant morbidity and provides no benefit for most breast cancer patients without axillary lymph node involvement. Surgical staging by axillary lymph node dissection is the usual practice in Nepal. Frozen section is not widely available. Ultrasound of the axilla can identify enlarged or abnormal nodes in up to half of the patients with breast cancer and FNAC or core biopsy of the nodes identifies up to $40 \%$ of patients with involved axillary nodes. ${ }^{32}$ Sentinel lymph node biopsy accurately assesses lymph node status and is associated with less morbidity than axillary dissection. ${ }^{33}$ Sentinel node mapping and biopsy are in the initial phase of use in Nepal. ${ }^{34,35}$ Breast reconstruction after mastectomy can produce good cosmetic results, especially where autologous tissue is used. Myocutaneous flaps using latissimus dorsi or transverse rectus abdominus muscles are increasingly used. ${ }^{36} \mathrm{Although}$ breast reconstruction plays a remarkable role in woman's physical, emotional and psychological recovery from breast cancer, immediate or delayed breast reconstruction is rarely performed in Nepal. However, chest wall reconstruction has been performed for breast cancer. ${ }^{37,38}$ 


\section{Radiation Therapy:}

Factors affecting the local recurrence include patient factors (e.g. young age), disease factors (e.g. extensive intraductal component, lymphovascular invasion and grade) and treatment factors (e.g. resection margins, intensity of radiotherapy and adjuvant systemic therapy). ${ }^{39}$ Radiotherapy has a crucial role in the control of postoperative breast cancer risk. There is a definite efficacy of breast conserving surgery and radiotherapy in terms of survival. Radiotherapy reduces the rate of local recurrence after breast conserving surgery and improves overall survival. ${ }^{40}$ Post-mastectomy radiotherapy is associated with a $66 \%$ reduction in risk of local recurrence. ${ }^{29}$ Moreover, there is a significant overall survival benefit associated with adjuvant therapy in those treated by mastectomy alone or mastectomy and axillary clearance. The addition of axillary irradiation post axillary lymph node dissection may be beneficial when it is likelihood of residual disease in the axilla. There is regional variation in the use of radiation therapy in Nepal. ${ }^{6}$

\section{Systemic Therapy:}

Breast cancer was the first malignancy for which effective systemic therapy was developed. ${ }^{41}$ Endocrine therapies are designed to counteract the proliferative effect of estrogen in estrogen receptor-positive breast cancer. Tamoxifen is currently the most widely used hormonal therapy. ${ }^{42}$ Tamoxifen-users require routine testing for endometrial evaluation. ${ }^{43}$ Aromatase inhibitors reduce estrogen production in postmenopausal women by inhibiting or inactivating an enzyme, aromatase. ${ }^{44}$ However, these inhibitors are ineffective in premenopausal women because they produce an increase in gonadotrophin secretion, which results in reduced feedback of estrogen. The selective estrogen receptor modulators such as aromatase inhibitors are also available in Nepal. However, these are expensive compared to Tamoxifen, the later is used even in postmenopausal women who cannot afford aromatase inhibitors.

There are many cytotoxic agents used in breast cancer either in post or pre operative settings. Anthracyclinebased chemotherapy regimen is commonly used in Nepal. ${ }^{45}$ Selection of adjuvant treatment is determined by prognostic factors. Preoperative chemotherapy increases the chance of BCS rather than mastectomy. ${ }^{46}$ Trastuzumab is a novel human monoclonal antibody that specifically targets HER-2 (predictive factor) and achieves good response in HER-2 positive breast cancer. ${ }^{47}$ Although trastuzumab, a very expensive molecule restricting its use to a selected group of patients, is available in Nepal.

\section{Palliative Care:}

Palliative care has a crucial role to improve the quality of life of women with metastatic breast disease. ${ }^{48}$ Pain is the most common and the most feared symptom to be controlled in cancer patients. There are special pain situations common in metastatic breast cancer. Pain secondary to bone metastasis is an alarming situation and needs specific management. Radiotherapy is the treatment of choice for the palliation of metastatic bone disease. ${ }^{49}$ Single fraction has been shown to be a very effective way in the achievement of pain control. Biphosphonates are recommended for routine breast cancer treatment in order to reduce the skeletal morbidity and also to control bone pain. ${ }^{50}$ Radiotherapy can be combined with early systemic therapy with biphosphonates to reduce morbidity of bone metastases. Palliative care services are in initial phases of development in Nepal. ${ }^{51}$

\section{Cancer Education:}

Most Nepalese women are not aware of the risk factors associated with breast cancer. Education status of the women in Nepal has a crucial value in the management of breast cancer. An educated woman understands her disease better and participates actively in the treatment process. Breast cancer awareness programs should be extended to the rural areas of Nepal where mass communication means are not adequate. Moreover, the educational difference between the patient and the specialized physician may create an atmosphere where there is little respect conveyed to the patient. Regular cancer education and appropriate trainings are important for treating physicians and nurses in order to improve the quality of service. ${ }^{52}$

In summary, cancer care is on the rise and breast cancer can be 'cured' even in Nepal. Cancer awareness particularly to women is the key factor for successful cancer control. Screening and early detection programs are extended throughout the country. A standard breast cancer treatment protocol should be followed by all the centers in order to measure the treatment outcomes. Although there are a few comprehensive cancer centers in Nepal, most of the centers are treating these cancer patients with limited resources. 


\section{REFERENCES}

1. Ferlay J, Bray F, Pisani P, Parkin DM. GLOBANCAN 2002. Cancer incidence, mortality and prevalence worldwide. IARC Cancer base. Lyon: IARC Press; 2004.5(2.0).

2. Pradhananga KK, Baral M, Shrestha BM. Multi-institution hospital-based cancer incidence data for Nepal: an initial report. Asian Pac J Cancer Prev. 2009;10:259-62.

3. Binu VS, Chandrashekhar TS, Subba SH, et al. Cancer pattern in Western Nepal: a hospital based retrospective study. Asian Pac J Cancer Prev. 2007;8:183-6.

4. World Health Organization. Disease specific NCD morbidity and mortality profile [Online]. 1998 [cited 2008 Feb 10]; [8 screens]. Available from: www.searo.who.int/LinksFiles/ NCD_Inforbase_disease-specific.pdf

5. Pisani P. Breast cancer: geographic variation and risk factors. J Envion Pathol Toxicol Oncol. 1992;11:313-6.

6. Bhattacharya S, Adhikari S. Evaluation of risk factors, diagnosis and treatment in carcinoma breast-a retrospective study. Kathmandu Univ Med J (KUMJ). 2006;4:54-60.

7. Singh Y, Sayami P, Sayami G, et al. Nepalese breast cancer in relation to reproductive factors: comparison between Nepalese and Japanese cases. Anticancer Res. 2002;22:319-23.

8. Vahabi M. Breast cancer screening methods: a review of the evidence. Health Care Women Int. 2003;24:773-93.

9. Tara S, Agrawal CS, Agrawal A. Validating breast self examination as screening modalities for breast cancer in eastern region of Nepal: a population based study. Kathmandu Univ Med J (KUMJ). 2008;6:89-93.

10. Sidhartha, Thapa B, Singh Y, Sayami P, Khanal U. Mammographic diagnosis of breast carcinoma: An institutional experience. J Nepal Med Assoc. 2008;47:62-5.

11. Stavros AT, Thickman D, Rapp CL, et al. Solid breast nodules: use of sonography to distinguish between benign and malignant lesions. Radiology. 1995;196:123-34.

12. Rahbar G, Sie AC, Hansen GC, et al. Benign versus malignant solid breast masses: US differentiation. Radiology. 1999;213:889-94

13. Irwig L, Houssami N, van Vliet $\mathrm{C}$, et al. New technologies in screening for breast cancer: a systemic review of their efficacy. Br J Cancer. 2004;90:2118-22.

14. Kampert JB, Whittemore AS, Paffenbarger RS Jr. Combined effect of child bearing, menstrual events and body size on agespecific breast cancer risk. Am J Epidemiol. 1988;128:962-79.

15. Prasad SN, Houserkova D. A comparison of mammography and ultrasonography in the evaluation of breast masses. Biomed Pap Med Fac Univ Palacky Olomouc Czech Repub. 2007;151:315-22

16. Berg WA, Gutierrez L, NessAiver MS, et al. Diagnostic accuracy of mammography, clinical examination, US and MR imaging in pre-operative assessment of breast cancer. Radiology. 2004;233:830-49.

17. Liberman L. Breast cancer screening with MRI - what are the data for patients for patients at high risk? N Engl J Med. 2004;351:497-500
18. Pradhan M, Dhakal H. Study of breast lump of 2246 cases by fine needle aspiration. JNMA J Nepal Med Assoc. 2008;47:205-9.

19. Ariga R, Bloom K, Reddy VB, et al. Fine-needle aspiration of clinically suspicious palpable breast masses with histopathologic correlation. Am J Surg. 2002;184:410-3.

20. Sayami P, Singh BM, Singh Y, et al. Retrospective analysis of breast cancer cases and surgical treatment in a period of ten years. JNMA J Nepal Med Assoc. 2001;40:112-9.

21. Shabot M, Goldberg I, Schick P, et al. Aspiration cytology is superior to Tru-cut needle biopsy in establishing the diagnosis of clinically suspicious breast masses. Ann Surg. 1982;196:122.

22. Matsumoto K, Sakamoto G, Nomura Y. International comparisons concerning breast cancer and steroid receptors. Anticancer Res. 1986;6:621-4.

23. Kaufman Z, Shpitz B, Shapiro M, Rona R, Lew S, Dinbar A. Triple approach in the diagnosis of dominant breast masses: combined physical examination, mammography, and fine needle aspiration. J Surg Oncol. 1994;56:254-7.

24. Ghimire B, Khan MI, Thapa B, Singh Y, Sayami. Accuracy of triple test score in the diagnosis of palpable breast lump. JNMA J Nepal Med Assoc. 2008;47:189-92.

25. Britton PD. Fine needle aspiration or core biopsy. Breast. 1999;8:1-4.

26. Parker SH, Jobe WE, Dennis MA, et al. US-guided automated large-core breast biopsy. Radiology. 1993;187:507-11.

27. Sharif K, Al-Ghazal SK, Blamey RW. Cosmetic assessment of breast conserving surgery for primary breast cancer. Breast. 1999;8:162-8.

28. Al-Ghazal SK, Fallowfield L, Blamey RW. Comparison of psychological aspects and patient satisfaction following breast conserving surgery, simple mastectomy and breast reconstruction. Eur J Cancer. 2000;36:1938-43.

29. Early Breast Cancer Trialists' Collaborative Group. Effects of radiotherapy and surgery in early breast cancer: an overview of the randomized trials. N Engl J Med. 1995;333:1444-55.

30. Morris AD, Morris RD, Wilson JF, et al. Breast conserving therapy versus mastectomy in early stage breast cancer: a metaanalysis of 10 year survival. Cancer J Sc Am. 1997;3:6-12.

31. Veronesi U, Galiberti V, Zurrida S, et al. Prognostic significance of number and level of axillary node metastases in breast cancer. Breast. 1993;2:224-8.

32. Kuenen-Boumeester V, Menke-Pluymers M, de Kanter AY, et al. Ultrasound-guided fine needle aspiration cytology of axillary lymph nodes in breast cancer patients: a preoperative staging procedure. Eu J Cancer. 2003;39:170-4.

33. Allweis TM, Badriyyah M, Bar Ad V, et al. Current controversies in sentinel lymph node biopsy for breast cancer. Breast. 2003;12:163-71.

34. Lamichhane N, Pradhan M, Neupane PR et al. Initial experience in a cancer hospital in Nepal with sentinel lymph node mapping and biopsy for evaluation of axillary lymph node status in palpable invasive breast cancer. Nepal Med Coll J. 2007;9:22-6. 
35. Lamichhane N, Shen KW, Li CL, et al. Sentinel lymph node biopsy in breast cancer patients after overnight migration of radiolabelled sulphur colloid. Postgrad Med J. 2004;80:546-50.

36. Khoo A, Kroll SS, Reece GP, et al. A comparison of resource costs of immediate and delayed breast reconstruction. Plast Reconstr Surg. 1998;101:964-8.

37. Thakur B, Bo TZ, Lamichhane N, Shrestha BM, Neupane PR, Dudani R. Retrospective study of chest wall tumors at a cancer hospital in Nepal. Nepal Med Coll J. 2005;7:39-42.

38. Tan ZB, Lamichhane N, Thakur B, Liu DX, Xiao QH. How we performed chest wall reconstruction: analysis 31 cases. Indian J Cancer. 2003;40:27-30.

39. Clarke DH, Li MG, Sarrazin D, et al. Analysis of local regional relapses in patients with early breast cancers treated by excision and radiotherapy: experience of the Institut GustaveRoussy. Int J Radiat Oncol Phys. 1985;11:137-45.

40. Touboul E, Buffat L, Belkacemi Y, et al. Local recurrences and distant metastases after breast conserving surgery and radiation therapy for early breast cancer.Int J Radiat Oncol Biol Phys. 1999;43:25-38.

41. Beatson JT. On the treatment of inoperable cases of carcinoma of the mamma: suggestions for a new method of treatment, with illustrative cases. Lancet. 1896;ii:104-7,162-5.

42. Mishra D, Poudel R, Kishore PV, Palaian S, Bista D, Mishra $P$. Interaction between warfarin and tamoxifen: a case report. Kathmandu Univ Med J (KUMJ). 2007;5:105-7.

43. Pokharel HP, Bhatla N, Kriplani A, Mukhopadhyay A, Srivastava A, Ralhan R. Evaluation of endometrial changes and p53 expression in tamoxifen treated women: comparison of various methods. Kathmandu Univ Med J (KUMJ). 2006;4:145-51.

44. Miller WR. Aromatase inhibitors: mechanism of action and role in the treatment of breast cancer. Semin Oncol. 2003;30:3-11.

45. Das RN, Poudel N. Could garlic be an useful adjuvant therapy in adriamycin heart failure? Kathmandu Univ Med J (KUMJ). 2006;4:337-9.

46. Makris A, Powels TJ, Ashley SE, et al. A reduction in the requirements for mastectomy in a randomized trial of neoadjuvant chemoendocrine therapy in primary breast cancer. Ann Oncol 1998;9:1179-84.

47. Sledge G. Advances in HER2-positive breast cancer. Clin Adv Hematol Oncol. 2008;6:98-100.

48. Higginson IJ, Findlay IG, Goodwin DM, et al. Is there evidence that palliative care teams alter end-of-life experiences of patients and their caregivers? J Pain Symptom Manage. 2003;25:150-68

49. Sze WM, Shelly MD, Held I, et al. Palliation of metastatic bone pain: single fraction versus multi-fraction radiotherapy. A systemic review of randomized trials. Clin Oncol. 2003;15:345-52.

50. Ross JR, Sauders Y, Edmonds P, Patel S, Broadley K, Johnston S. Systemic review of the role of biphosphonates on skeletal morbidity in metastatic breast cancer. Br Med J. 2003;327:469.

51. Brown S, Black F, Vaidya P, Shrestha S, Ennals D, LeBaron VT. Palliative care development: the Nepal model. J Pain Symptom Manage. 2007;33:573-7.

52. Braun CM, Itano JK. Cancer care in Nepal: variables that affect diagnosis, treatment, and prognosis: a case study. Cancer Nurs. 2001;24:137-42. 